\title{
VALOR PRONÓSTICO DE BIOMARCADORES EN SEPSIS. HOSPITAL LUIS VERNAZA, ENERO A DICIEMBRE DE 2015
}

\author{
PROGNOSTIC VALUE OF BIOMARKERS IN SEPSIS. LUIS VERNAZA HOSPITAL, JANUARY TO
}

DECEMBER 2015

VALOR PROGNÓSTICO DE BIOMARCADORES NA SEPSE. HOSPITAL DE LUIS VERNAZA DE JANEIRO A DEZEMBRO

DE 2015

\section{KAREN LISSETTE HANZE VILLAVICENCIO}

Universidad Católica de Santiago de Guayaquil, Guayaquil, Ecuador

\section{Resumen}

Introducción: la sepsis es una condición compleja que tiene un periodo prodrómico corto así como una alta mortalidad a corto plazo. Se necesitan marcadores lo suficientemente específicos y sensibles que ayuden a predecir mortalidad para esta entidad. Objetivo: establecer el rol de los biomarcadores en la evolución de la sepsis en el hospital Luis Vernaza, desde 01 enero hasta 31 de diciembre de 2015. Materiales y métodos: estudio observacional retrospectivo de 165 pacientes con diagnóstico de sepsis a los que se les midió procalcitonina, proteína c reactiva y conteo de leucocitos en las primeras 24 horas. Resultados: tanto los niveles de procalcitonina $(p=0,316)$ como los de proteína $c(p=0,099)$ no mostraron diferencias significativas entre los no sobrevivientes y sobrevivientes. Conclusiones: los biomarcadores no mostraron valor pronóstico de mortalidad por sepsis por lo que se sugiere futuras investigaciones.

PALABRAS CLAVE: sepsis, biomarcadores, pronóstico, mortalidad.

Abstract

Introduction: sepsis is a complex condition that has a short prodromal period as well as a high short-term mortality. Specific and sensitive enough markers are needed to help predict mortality for this entity. Objective: to establish the role of biomarkers in the evolution of sepsis in the Luis Vernaza Hospital, from January 01 to December 31, 2015. Materials and methods: retrospective observational study of 165 patients diagnosed with sepsis to who we measured procalcitonin, $c$-reactive protein and leukocyte count in the first 24 hours. Results: both procalcitonin $(p=0.316)$ and $c$-reactive protein $(p=0.099)$ levels showed no significant differences between non-survivors and survivors. Conclusions: the biomarkers did not show to be of prognostic value for mortality due to sepsis, so further investigations are suggested.

KEYWORDS: sepsis, biomarkers, prognosis, mortality.

Resumo

Introdução: a sepse é uma condição complexa, que tem com curto período prodrômico e uma alta mortalidade a curto prazo. Se precisam marcadores suficientemente específicos e sensível que ajudem a prever a mortalidade para esta entidade. Objetivo: estabelecer o papel dos biomarcadores na evolução na sepse no hospital Luis Vernaza, desde 01 janeiro até 31 de dezembro de 2015. Materiais e métodos: estudo observacional retrospectivo de 165 pacientes com diagnostico na sepse aqueles que foram medidos Procalcitonina, proteína c reativa e contagem de leucócitos nas primeiras 24 horas. Resultados: os níveis de Procalcitonina $(\mathrm{p}=0,316)$ e proteína c reativa $(\mathrm{p}=0,099)$ não mostraram diferenças significativas entre nos sobreviventes. Conclusões: os biomarcadores não mostrarem valor pra prever mortalidade por sepse pelo que se sugere futuras pesquisas

PALABRAS-CHAVE: sepse, biomarcadores, prognóstico, mortalidade. 
INTRODUCCIÓN

La sepsis es una desrregulación bioquímica y fisiológica a las infecciones que son responsables por la alta mortalidad en los pacientes críticos, lo cual aumenta los gastos hospitalarios anuales. ${ }^{1,2}$ Se estima que en Estados Unidos de Norteamérica la mortalidad por sepsis llega a ser del $30 \%$ y en sepsis severa del $50 \%$. Aproximadamente la mitad se desarrolla fuera de la Unidad de Cuidados Intensivos (UCI), llegando a morir un cuarto de los casos, mientras que los que desarrollan shock séptico dentro de la terapia intensiva tienen una tasa de mortalidad del 80\% ${ }^{2-4}$ En Ecuador, la base de datos del Instituto Nacional de Estadística y Censos (INEC) del 2013 elaborado por el Ministerio de Salud Pública (MSP), establece que de un total de 63.104 defunciones, la mortalidad por septicemia fue un total de 398 (tasa de mortalidad de $0,63 \%) .{ }^{5}$ Sin embargo, no existen datos que nos indiquen el porcentaje de mortalidad global por sepsis, porcentaje de aquellos casos que evolucionan a sepsis severa o los que fallecen antes de ingresar a la terapia intensiva por shock séptico.

Conocer la mortalidad hospitalaria global de sepsis y saber utilizar correctamente los recursos de laboratorio, sería de suma importancia para disminuir los gastos hospitalarios y optimizar su uso para el diagnóstico de sepsis. ${ }^{6}$ Simultáneamente, al reducir la mortalidad, se disminuye la discapacidad y deterioro neurocognitivo que se producen como secuelas de una sepsis severa o shock séptico en los pacientes que superan el cuadro. El presente trabajo tiene como objetivo principal establecer el rol de los biomarcadores en el pronóstico de sepsis, esto evidenciará la sensibilidad y especificidad de los marcadores y de esta forma implementar su uso en la práctica clínica.

\section{MATERIALES Y MÉTODOS}

El presente estudio tiene un diseño retrospectivo, observacional, descriptivo y de corte transversal. Fue realizado en el hospital Luis Vernaza desde enero a diciembre 2015. Se seleccionó como población de estudio a los pacientes adultos con diagnóstico de sepsis, sepsis severa y/o shock séptico, al momento del ingreso o durante su estancia hospitalaria con un periodo de hospitalización superior a 24 horas de acuerdo a la definición de ACCP/SCCM 2001. Se prescindieron para este estudio pacientes menores de 18 años, mujeres con sospecha o confirmación de embarazo, diagnóstico de pancreatitis aguda, quemaduras y con datos de inclusión incompletos. Los datos fueron adquiridos mediante la revisión de las historias clínicas de los pacientes. Las siguientes variables fueron recolectadas: edad, género, comorbilidad, foco de infección, mortalidad intrahospitalaria, estancia hospitalaria, diagnósticos de ingreso y de egreso, germen aislado por cultivo microbiológico, títulos de proteína $C$ reactiva, procalcitonina, conteo completo de glóbulos blancos, puntaje APACHE II y puntaje SOFA. En cuanto a los títulos de procalcitonina y proteína c reactiva, se aseguró que fueran muestras tomadas las primeras 24 horas del diagnóstico de sepsis para considerar la inclusión del paciente. Se extrajo $5 \mathrm{ml}$ de sangre venosa, se la mantuvo a temperatura ambiente por 10-15 min hasta la formación del coágulo y luego se la centrifugó por 10 minutos a 3500 rpm; los niveles de la procalcitonina fueron medidos del suero restante mediante electroquimioluminiscencia (ECLIA) por el analizador inmunológico Cobas e 601 Roche Elecsys BRAHMS PCT, mientras que la proteína c reactiva mediante espectrofotometría por el analizador bioquímico Cobas c 501 Roche.

La mortalidad en la UCI fue registrada y los días de hospitalización se documentaron desde la fecha de ingreso al hospital hasta de egreso, en caso de alta médica o muerte. Si faltaban datos de laboratorio para el cálculo del puntaje SOFA y/o APACHE, aquel paciente quedaba excluido.

Los datos recolectados fueron ingresados en una hoja de cálculo de Microsoft Office Excel 2010 donde se procedió a su tabulación, para luego ser analizados por el programa SPSS Statistics (IBM corporation, EEUU) con sus respectivos gráficos. Para el análisis de las variables cualitativas, se utilizó frecuencias simples, porcentajes y sus relaciones se las calculó con Chi2, mientras que para las variables cuantitativas se utilizó la media, desviación estándar y T de student.

Se obtuvo la aprobación del Departamento de Investigación, del Comité Científico y de Bioética del Hospital; por su carácter retrospectivo observacional se prescindió del consentimiento informado y se siguió las recomendaciones de la declaración de Helsinki.

\section{RESULTADOS}

Para la realización del presente trabajo se incluyeron inicialmente 165 pacientes que cumplían los criterios de SIRS de acuerdo a la definición de ACCP/SCCM 2001, se excluyeron 65 pacientes que presentaban algún criterio de exclusión, por 
lo que un total de 100 pacientes fue la población final del estudio. Los pacientes fueron manejados por los servicios de medicina interna y Cirugía General. Se procedió a estudiar el foco infeccioso de acuerdo al sistema afecto: piel y parte blandas, pulmonar, cardiaco, abdominal, nefro-urológico y neurológico. La edad promedio fue $64,18 \pm$ 18,15 de los cuales $59 \%$ fueron femeninos y $41 \%$ masculinos. La principal comorbilidad que presentaron los pacientes fue hipertensión arterial (41\%) seguido de diabetes mellitus (31\%). Los dos grupos presentaron características similares sin diferencias significativas, a excepción del género en el que la mayoría de la mortalidad recayó en el femenino $(45 \%$ vs $14 \%$; $p=0,001)$ con un promedio de edad de 66 años $(65,69 \pm 17,80$ vs $56,25 \pm 18,48$; $\mathrm{p}<0,05)$. Las características basales y resultados de los pacientes se presentan en la tabla 1.

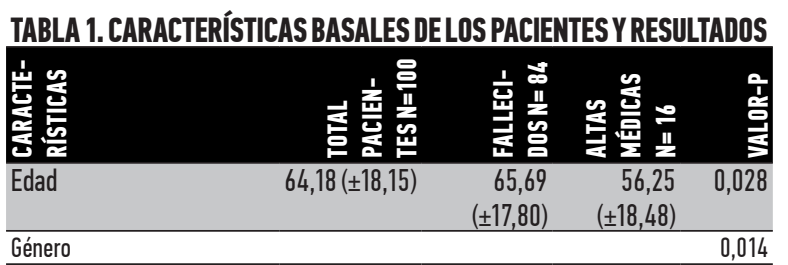

\begin{tabular}{llllr} 
Género & & & & 0,014 \\
\hline Femenino (\%) & 59 & 45 & 14 & - \\
\hline Masculino (\%) & 41 & 39 & 2 & - \\
\hline
\end{tabular}

Comorbilidades

\begin{tabular}{lrrrr}
\hline Hipertensión arterial & 41 & 34 & 7 & 0,807 \\
\hline Diabetes Mellitus & 31 & 29 & 2 & 0,081 \\
\hline Cirugía previa & 7 & 5 & 2 & 0,347
\end{tabular}

\begin{tabular}{|c|c|}
\hline Foco infeccioso & 0,166 \\
\hline
\end{tabular}

\begin{tabular}{lrrrrr}
\hline Pulmonar & 15 & 14 & 1 & - \\
\hline Abdominal & 52 & 45 & 7 & - \\
\hline Nefro-urológico & 13 & 8 & 5 & - \\
\hline Piel y partes blandas & 10 & 8 & 2 & - \\
\hline Otros & 10 & 9 & 1
\end{tabular}

Otros

\begin{tabular}{lr} 
Tipo de manejo & 0,609 \\
\hline
\end{tabular}

\begin{tabular}{llllr}
\hline Clínico & 45 & 36 & 9 & - \\
\hline Quirúrgico & 38 & 33 & 5 & - \\
\hline Clínico y quirúrgico & 17 & 15 & 2 & -
\end{tabular}

Clínico y quirúrgico

\begin{tabular}{llllr}
\hline Cultivo positivo & 68 & 56 & 12 & -
\end{tabular}

\begin{tabular}{lrrrr}
\hline Cultivo negativo & 32 & 28 & 4 & - \\
\hline Estancia hospitalaria & $30,52( \pm 35,45)$ & 28,85 & 39,31 & 0,288
\end{tabular}

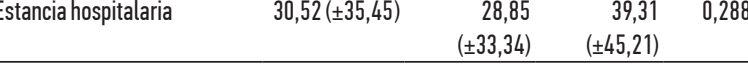

\begin{tabular}{llrrr}
\hline Procalcitonina (día 1) & 27,8 & 26,00 & 37,26 & 0,316
\end{tabular}

$(21,15-34,46)(18,88-33,13)(16,37-58,14)$

\begin{tabular}{lrrrr}
\hline Proteína C Reactiva (día 1) & 187,86 & 177,57 & 241,92 & 0,099 \\
& $(165,62-$ & $(152,70-$ & $(192,31-$ & \\
& $210,11)$ & $202,44)$ & $291,53)$ & \\
\hline Leucocitos totales (día 1) & 19096 & 18874,05 & 20261,88 & 0,745 \\
& $(16784-21408)$ & $(16286,15-$ & $(14188,09-$ & \\
& & $21461,95)$ & $26335,66)$ & \\
\hline SOFA score (día 1) & 9,61 & 9,96 & 7,75 & 0,182 \\
& $(8,92-10,30)$ & $(9,21-10,72)$ & $(5,99-9,51)$ & \\
\hline APACHE Il score (día 1) & 18,42 & 18,95 & 15,63 & 0,273 \\
& $(17,16-19,68)$ & $(17,54-20,37)$ & $(12,83-18,42)$ & \\
\hline
\end{tabular}

Valores expuestos como la media junto a $\pm \mathrm{DS}$ (desviación estándar) $0 \mathrm{n}$ (porcentaje) e IC (intervalo de confianza); APACHE: Acute physiology and chronic health evaluation; SOFA: Sequential organ function assessment.
Al momento del ingreso por emergencia, se estableció el diagnóstico de sepsis en 80 pacientes, sepsis severa en 13 y shock séptico en 7. Durante su estancia hospitalaria la evolución clínica mostró que en el grupo de sepsis, 6 pacientes evolucionaron a sepsis severa, 34 a shock séptico y 37 a falla multiorgánica; del grupo de sepsis severa, 10 desarrollaron falla multiorgánica y 3 shock séptico, mientras que del grupo de shock séptico, 3 permanecieron en su actual condición y 4 evolucionaron a falla multiorgánica $(\mathrm{p}<0,001)$, (tabla 2), 45 paciente fueron tratados clínicamente, 38 necesitaron resolución quirúrgica mientras que en 17 el manejo fue en conjunto de las dos especialidades. Del total de pacientes fallecieron 84 y 16 fueron dados de alta médica.

\begin{tabular}{|c|c|c|c|}
\hline \multirow{2}{*}{$\begin{array}{l}\text { DIAGNÓSTICO DE } \\
\text { EGRESO }\end{array}$} & \multicolumn{2}{|c|}{ MORTALIDAD } & \multirow[t]{2}{*}{ VALOR-P } \\
\hline & FALLECIDOS & ALTAS MÉDICAS & \\
\hline Chi2 & $0(0 \%)$ & $3(100 \%)$ & \multirow{5}{*}{$<0,001$} \\
\hline Sepsis severa & $4(66,7 \%)$ & $2(33,3 \%)$ & \\
\hline Shock séptico & $31(77,5 \%)$ & $9(22,5 \%)$ & \\
\hline Falla multiorgánica & $49(96 \%)$ & $2(4 \%)$ & \\
\hline Total & 84 & 16 & \\
\hline
\end{tabular}

Valores expuestos como n (porcentaje).

El principal foco de infección fue el abdominal (52\%) seguido del pulmonar (15\%), nefro-urológico $(13 \%)$ e infecciones de piel y partes blandas $(10 \%)$. El 68\% de los cultivos biológicos proporcionaron un resultado positivo, siendo el hemocultivo el de mayor prevalencia (34\%); se aislaron principalmente gérmenes gram negativos (63\%) de los cuales los del género Escherichia y Klebsiella, fueron los más comunes. (Tabla 3).

TABLA 3. CARACTERÍSTICAS MICROBIOLÓGICAS (CHI2 VALOR P=0,032)

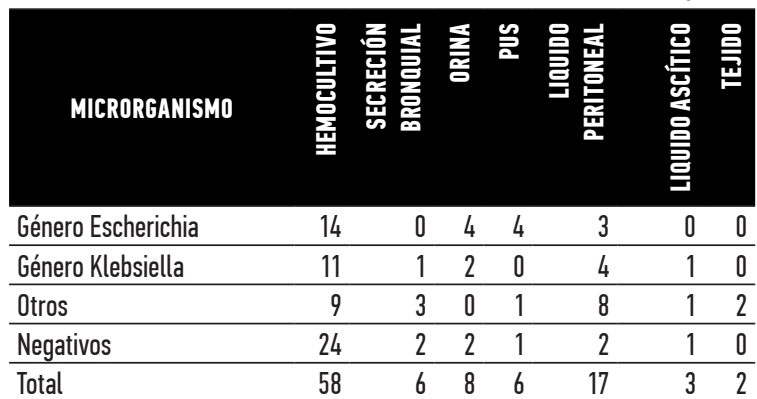

Los biomarcadores no presentaron diferencias significativas entre el grupo de fallecidos y de sobrevivientes. Tanto los niveles de procalcitonina $[26,00(18,88-33,13)$ vs $37,26(16,37-58,14) ; p=0,316]$ 
como los de proteína c reactiva $[177,57(152,70-$ $202,44)$ vs $241,92(192,31-291,53) ; p=0,099]$ no mostraron diferencias significativas entre los dos grupos. Los niveles de leucocitos totales fueron los menos significativos ( $\mathrm{p}=0,745)$. Resultados similares presentan los puntajes de severidad y fallo orgánico secuencial, APACHE II y SOFA score respectivamente.

Asimismo se estableció una relación entre los niveles de los biomarcadores procalcitonina, proteína c reactiva y leucocitos totales con la mortalidad. Se observó que el grupo de pacientes con valores de procalcitonina entre 10 - $50 \mathrm{ng} /$ $\mathrm{mL}$, obtuvo la mayor cantidad de fallecidos $(95,8 \%$ vs $4,2 \%)$, seguido de los pacientes con valores de $<2 \mathrm{ng} / \mathrm{mL}$, en los que un $92 \%$ vs $8,3 \%$ fallecieron $(\mathrm{p}=0,078)$.

Por otro lado, los pacientes con valores de proteína c reactiva $<100 \mathrm{mg} / \mathrm{L}$, un $95,8 \%$ fallecieron; mientras que un $4,2 \%$ fueron dados de alta médi$\mathrm{ca}(\mathrm{p}=0,138)$ y entre los pacientes con leucocitos $<10.000 \mathrm{cel} / \mathrm{mm}^{3}$ fallecieron $85 \%$, comparado con los sobrevivientes $15 \%(p=0,991)$. Los valores se detallan en la tabla 4.

TABLA 4. RELACIÓN ENTRE BIOMARCADORES Y MORTALIDAD

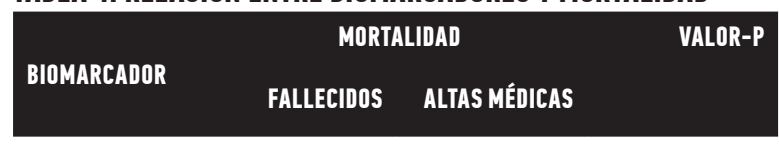

$\begin{array}{ll}\text { Chi2 } & 0,078\end{array}$

\begin{tabular}{lrrr}
\hline$<2 \mathrm{ng} / \mathrm{mL}$ & 91,7 & 8,3 & - \\
\hline $2-10 \mathrm{ng} / \mathrm{mL}$ & 76,9 & 23,1 & - \\
\hline $10-50 \mathrm{ng} / \mathrm{mL}$ & 95,8 & 4,2 & - \\
\hline$>50 \mathrm{ng} / \mathrm{mL}$ & 73,1 & 26,9 & - \\
\hline Proténa C reactiva & & & 0,138
\end{tabular}

\begin{tabular}{llll}
\hline$<100 \mathrm{mg} / \mathrm{L}$ & 95,8 & 4,2 & - \\
\hline $100-200 \mathrm{mg} / \mathrm{L}$ & 83,8 & 16,2 & - \\
\hline$>200 \mathrm{mg} / \mathrm{L}$ & 76,9 & 23,1 & -
\end{tabular}

Leucocitos totales $\quad 0,991$

\begin{tabular}{llll}
\hline$<10.000 \mathrm{cel} /$ & 85 & 15 &
\end{tabular}

$\mathrm{mm} 3$

$10.000-20.000 \quad 83,7 \quad 16,3$

$\mathrm{cel} / \mathrm{mm} 3$

$\begin{array}{llll}>20.000 \mathrm{cel} / & 83,8 & 16,2 \\ \mathrm{~mm} 3\end{array}$

TOTAL

TOTAL

84

16

Valores expuestos como porcentajes.

La relación presentada entre los Biomarcadores y la estancia hospitalaria evidenció que niveles de procalcitonina entre $2-10 \mathrm{ng} / \mathrm{mL}$ presentó estancias hospitalarias hasta de 30 días (58\%), mientras que los niveles entre $10^{-}-50 \mathrm{ng} / \mathrm{mL}$ tenían la estancia más corta con el $25 \%$ de pacientes menos de 10 días. Los pacientes con niveles de proteína c reactiva $<100 \mathrm{mg} / \mathrm{L}$ presentaron así mismo una estancia hospitalaria entre 10-30 días (46\%) y aquellos con niveles entre 100-200 $\mathrm{mg} / \mathrm{L}$ permanecieron menos de 10 días en el ámbito hospitalario (30\%).

Finalmente los pacientes con leucocitos $>20.000$ $\mathrm{cel} / \mathrm{mm} 3$ tuvieron la estancia entre 10-30 días $(46 \%)$ mientras que el grupo con leucocitos $<10000$ $\mathrm{cel} / \mathrm{mm} 3$ presentaba estancia más corta $(40 \%)$. No se encontró relación estadísticamente significativa entre la mortalidad y estancia hospitalaria $\mathrm{y}$ los niveles de los biomarcadores ( $\mathrm{p}=1,00)$. ). Los valores se detallan en la tabla 5.

TABLA 5. RELACIÓN ENTRE BIOMARCADORESY ESTANCIA HOSPITALARIA

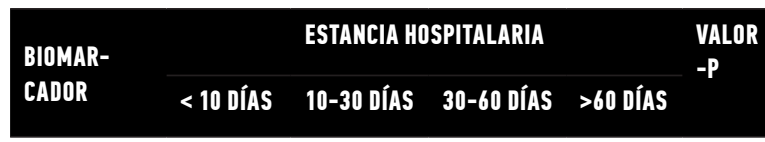

\begin{tabular}{|c|c|c|c|c|c|}
\hline \multicolumn{5}{|l|}{ Chi2 } & \multirow{2}{*}{$\begin{array}{l}0,287 \\
-\end{array}$} \\
\hline$<2 \mathrm{ng} / \mathrm{mL}$ & 25 & 33,3 & 37,5 & 4,2 & \\
\hline $2-10 \mathrm{ng} / \mathrm{mL}$ & 34,6 & 23,1 & 26,9 & 15,4 & - \\
\hline $10-50 \mathrm{ng} / \mathrm{mL}$ & 16,7 & 58,3 & 12,5 & 12,5 & - \\
\hline$>50 \mathrm{ng} / \mathrm{mL}$ & 26,9 & 42,3 & 19,2 & 11,5 & - \\
\hline \multicolumn{5}{|c|}{ Proteína $C$ reactiva } & 0,857 \\
\hline$<100 \mathrm{mg} / \mathrm{L}$ & 20,8 & 45,8 & 16,7 & 16,7 & - \\
\hline $\begin{array}{l}100-200 \\
\mathrm{mg} / \mathrm{L}\end{array}$ & 29,6 & 35,1 & 27 & 8,1 & - \\
\hline$>200 \mathrm{mg} / \mathrm{L}$ & 25,6 & 38,5 & 25,6 & 10,3 & - \\
\hline \multicolumn{5}{|c|}{ Leucocitos totales } & 0,726 \\
\hline $\begin{array}{l}<10.000 \mathrm{cel} / \\
\mathrm{mm} 3\end{array}$ & 40 & 30 & 20 & 10 & - \\
\hline $\begin{array}{l}10.000- \\
20.000 \mathrm{cel} / \\
\mathrm{mm} 3\end{array}$ & 23,3 & 37,2 & 25,6 & 14 & - \\
\hline $\begin{array}{l}>20.000 \mathrm{cel} / \\
\mathrm{mm} 3\end{array}$ & 21,6 & 45,9 & 24,3 & 8,1 & - \\
\hline TOTAL & 26 & 39 & 24 & 11 & \\
\hline
\end{tabular}

Valores expuestos como porcentajes.

A pesar que no evidenciaron diferencias significativas entre los grupos, el APACHE II y el SOFA score mostraron un AUC $>0,5[0,6$ vs 0,7 (IC $0,5-0,8$ vs $0,6-0,8) ; p=0,06$ vs $p=0,01$ ] respectivamente, por lo que se plantea que estos puntajes de severidad tienen una buena capacidad para predecir mortalidad por sepsis, con el punto de corte 10,50 y 5,50 , respectivamente como se aprecia en la figura 1. 


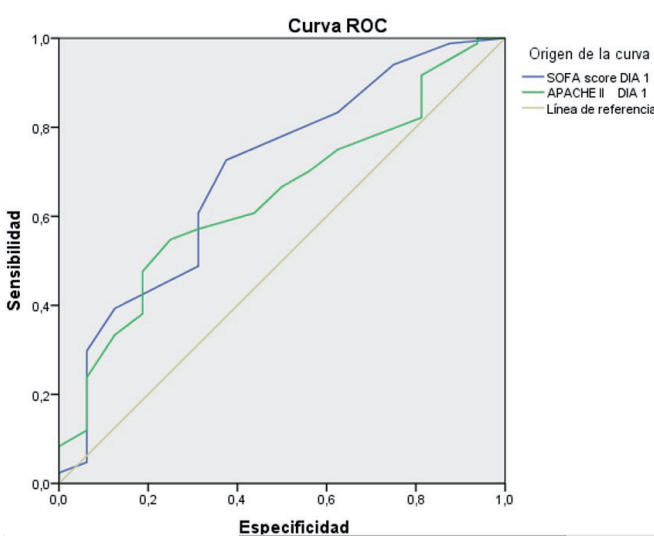

\begin{tabular}{lrr}
\hline PARÁMETROS & APACHE II & SOFA \\
\hline Sensibilidad & $91,7 \%$ & $94 \%$ \\
\hline Especificidad & $81,3 \%$ & $75 \%$ \\
\hline AUC & 0,644 & 0,699 \\
\hline Valor-p Chi2 & 0,069 & 0,012
\end{tabular}

Figura 1. Curva ROC para análisis de sensibilidad y especificidad del APACHE Il y SOFA score como predictores de mortalidad.

\section{DISCUSIÓN}

A pesar de un mejor conocimiento global de las complicaciones y secuelas ocasionadas por sepsis, aún no se logra disminuir la mortalidad a corto plazo de manera significativa. En el presente estudio la mortalidad por sepsis fue alta, de los cuales aproximadamente la mitad de todos los casos evolucionaron a falla multiorgánica. La mortalidad global encontrada es consistente con lo que se ha reportado en otros estudios; de igual manera la relación directa, entre la mayor mortalidad y la severidad del cuadro de sepsis. ${ }^{12,13} \mathrm{El}$ aumento de los biomarcadores está en relación directa y proporcional a la severidad del cuadro séptico y la disfunción orgánica; consecuentemente, se podría establecer una relación también directa entre la mortalidad y los altos niveles de estos. ${ }^{12,13}$ sin embargo, al valorar los tres marcadores sanguíneos individualmente, para apreciar el valor pronóstico de cada uno en la mortalidad por sepsis, no se encontró diferencias significativas, tanto en los valores de procalcitonina, proteína C reactiva y de leucocitos totales entre fallecidos y las altas médicas. El presente estudio reveló que los marcadores por separado, no brindan una certeza diagnóstica ni estaban relacionados con la mortalidad por sepsis ni con la estancia hospitalaria. Estos resultados, hasta cierto punto, discrepan de estudios previos en los que se ha demostrado la asociación entre niveles elevados de procalcitonina con la mortalidad a corto plazo y a los 28 días. ${ }^{14-16}$ Jain et al. (2014) determinaron que existe una diferencia significativa de los valores de PCT entre los no sobrevivientes y sobrevivientes. ${ }^{15}$
Se demostró en el estudio prospectivo de Iwashyna et al (2010), que si se compara el estado cognitivo y funcional de los pacientes justo antes y después del cuadro de sepsis severa, aumenta la prevalencia en un $16.7 \%$ del deterioro cognitivo a largo plazo (95\% CI, 13.8\%-19.7\%) (P<.001). ${ }^{7}$ Por lo tanto, la sepsis es a menudo un evento centinela en la salud de los adultos mayores, que se traducirá en descensos progresivos de su nivel neurocognitivo, que tendrá repercusiones a largo plazo, independientemente de las repercusiones hacia los familiares y las instituciones que se encargan de su cuidado, además de las implicaciones socioeconómicas que representa.

De acuerdo al estudio de Hegazy MA et al. (2014) la búsqueda de marcadores que disminuyan la morbimortalidad por infecciones es necesario, ya que la clínica de sepsis y de SRIS no infeccioso es muy similar. ${ }^{8}$ Entre estos marcadores, la proteína c reactiva muestra capacidad para diagnosticar el estado de septicemia y su evolución a sepsis seve$\mathrm{ra}$, mientras que las variaciones de procalcitonina predicen la evolución a shock séptico y disfunción multiorgánica. ${ }^{9-11}$

Los puntajes de severidad, APACHE-II y SOFA score, mostraron tener buena capacidad para indicar evolución desfavorable del cuadro infeccioso, como evidenciaron Ruiz-Álvarez et al. (2008), que estos sistemas de clasificación de severidad se relacionaron independientemente al diagnóstico de infección y los pacientes con puntajes altos de SOFA, fallecieron a corto plazo. ${ }^{17,18}$ En este estudio únicamente el puntaje SOFA demostró tener la capacidad de predecir mortalidad con $94 \%$ de sensibilidad y $75 \%$ de especificidad ( $\mathrm{p}=0,01$ ).

\section{LIMITACIONES DEL ESTUDIO}

La mayor limitación del estudio es su carácter retrospectivo y por ende la necesidad de depender de datos que se habían obtenido previamente, tal vez de manera no tan exhaustiva y personalizada como hubiera ofrecido un estudio de carácter prospectivo, dirigido específicamente a establecer las relaciones de estos biomarcadores con la mortalidad y la estancia hospitalaria. Al momento de la recolección de datos, se dificultó interpretar los escenarios en los que se diagnosticaba y manejaban los cuadros de sepsis. Además, es muy importante recalcar la inexistencia de un protocolo para el uso de estos marcadores en emergencia y en la unidad de terapia intensiva, por lo que varios sujetos que podrían haber estado incluidos en el estudio, quedaron excluidos por tener datos de laboratorio 
incompletos. En lo que respecta al cálculo de los puntajes de severidad, en la gran mayoría de los sujetos no constaban en la historia clínica, por lo que hubo la necesidad de calcularlos de acuerdo a los datos consignados en las mismas.

\section{CONCLUSIONES}

A pesar de que estos biomarcadores no mostraron relación con la mortalidad, la PCT y la PCR deberían seguir utilizándose como auxiliar diagnóstico para descartar sepsis en lugar de diagnosticarla. Futuras investigaciones de carácter prospectivo, con grupos control a los que se les mida consecutivamente los valores de PCR y/o PCT con protocolos predeterminados, se necesitan para confirmar estos resultados.

\section{RECOMENDACIÓN}

Aunque se emplean nuevas estrategias para una mejor estratificación de los pacientes en emergencia, esto no es suficiente y la mortalidad se mantiene en los mismos porcentajes. Se necesitan mejores auxiliares diagnósticos que apoyen el diagnóstico y ofrezcan un mejor panorama de la severidad del cuadro. Es primordial determinar las ventajas y desventajas de los biomarcadores antes de implementarlos en la práctica clínica rutinaria. Se recomiendan futuras investigaciones para validar estos resultados.

\section{REFERENCIAS BIBLIOGRÁFICAS}

1. Levy MM, Fink MP, Marshall JC, Abraham E, Angus D, Cook D, et al. 2001 SCCM/ESICM/ACCP/ATS/SIS International Sepsis Definitions Conference. Intensive Care Med. 2003 Mar 28; 29(4):530-8.

2. E, Angus D, Cook D, et al. 2001 SCCM/ESICM/ACCP/ ATS/SIS International Sepsis Definitions Conference. Intensive Care Med. 2003 Mar 28; 29(4):530-8.

3. Angus DC, Linde-Zwirble WT, Lidicker J, Clermont G, Carcillo J, Pinsky MR. Epidemiology of severe sepsis in the United States: analysis of incidence, outcome, and associated costs of care. Crit Care Med 2001; 29: 1303-10 doi: 10.1097/00003246-200107000-00002 pmid: 11445675 .

4. Mayr FB, Yende S, Angus DC. Epidemiology of severe sepsis. Virulence. 2014 Jan 1; 5(1):4-11.

5. Información estadística y geográfica de salud [Internet]. Ministerio de Salud Pública. [cited 2015 Jun 12]. Available from: http://www.salud.gob.ec/ informacion-estadistica-de-produccion-de-salud/

6. Cheng AC, West TE, LimmathurotsakulD, Peacock SJ. Strategies to Reduce Mortality from Bacterial Sepsis in Adults in Developing Countries. PLoS Medicine. 2008 Aug 19; 5(8):e175.
7. Iwashyna TJ, Ely EW, Smith DM, Langa KM. Longterm cognitive impairment and functional disability among survivors of severe sepsis. Jama. 2010; 304(16):1787-94.

8. Hegazy MA, Omar AS, Samir N, Moharram A, Weber S, Radwan WA. Amalgamation of procalcitonin, Creactive protein, and sequential organ failure scoring system in predicting sepsis survival. Anesth Essays Res. 2014; 8(3):296-301.

9. Sankar V, Webster NR. Clinical application of sepsis biomarkers. Journal of Anesthesia. 2013 Apr; 27(2):269-83.

10. Nargis W, Ibrahim M, Ahamed BU. Procalcitonin versus C-reactive protein: Usefulness as biomarker of sepsis in ICU patient. Int J Crit Illn Inj Sci. 2014; 4(3):195-9.

11. Silva BN, Andriolo RB, Salomão R, Atallah ÁN. Effectiveness and safety of procalcitonin evaluation for reducing mortality in adult patients with sepsis, severe sepsis and septic shock. Cochrane Database of Systematic Reviews 2014, Issue 2. Art. No.: CD010959. DOI: 10.1002/14651858.CD010959

12. Jawad I, Lukšić I, Rafnsson SB. Assessing available information on the burden of sepsis: global estimates of incidence, prevalence and mortality. Journal of global health [Internet]. 2012 [cited 2015 Oct 28]; 2(1). Available from: http://www.ncbi.nlm.nih.gov/pmc/ articles/PMC3484761/

13. Adhikari NK, Fowler RA, Bhagwanjee S, Rubenfeld GD. Critical care and the global burden of critical illness in adults. The Lancet. 2010; 376(9749):1339-46.

14. Hoeboer SH, Groeneveld ABJ. Changes in Circulating Procalcitonin Versus $C$ - reactive protein in Predicting Evolution of Infectious Disease in Febrile, Critically Ill Patients. Stover CM, editor. PLoS ONE. 2013 Jun 6; 8(6):e65564.

15. Jain S, Sinha S, Sharma SK, Samantaray JC, Aggrawal P, Vikram NK, et al. Procalcitonin as a prognostic marker for sepsis: a prospective observational study. BMC research notes. 2014; 7(1):458.

16. Póvoa P, Teixeira-Pinto AM, Carneiro AH. Portuguese Community-Acquired Sepsis Study Group SACiUCI. C-reactive protein, an early marker of communityacquired sepsis resolution: a multi-center prospective observational study. Critical Care. 2011; 15(4):R169.

17. Prkno A, Wacker C, Brunkhorst FM, Schlattmann P. Procalcitonin-guided therapy in intensive care unit patients with severe sepsis and septic shock-a systematic review and meta-analysis. Crit Care. 2013; 17(6):R291.

18. Ruiz-Alvarez MJ, Garcia-Valdecasas S, De Pablo R, Sanchez Garcia M, Coca C, Groeneveld TW, et al. Diagnostic Efficacy and Prognostic Value of Serum Procalcitonin Concentration in Patients With Suspected Sepsis. Journal of Intensive Care Medicine. $2008 \mathrm{Nov}$ 25; 24(1):63-71. 